\title{
InGaZnO TFT behavioral model for IC design
}

\author{
Pydi Bahubalindrun $^{1} \cdot$ Vítor Tavares $^{2} \cdot$ Pedro Barquinha $^{1} \cdot$ Pedro Guedes de Oliveira $^{2}$. \\ Rodrigo Martins ${ }^{1} \cdot$ Elvira Fortunato ${ }^{1}$
}

Received: 25 September 2015/Revised: 12 February 2016/Accepted: 15 February 2016/Published online: 26 February 2016

(C) Springer Science+Business Media New York 2016

\begin{abstract}
This paper presents a behavioral model for amorphous indium-gallium-zinc oxide thin-film transistor using artificial neural network (ANN) based equivalent circuit (EC) approach to predict static and dynamic behavior of the device. In addition, TFT parasitic capacitances $\left(\mathrm{C}_{\mathrm{GS}}\right.$ and $\left.\mathrm{C}_{\mathrm{GD}}\right)$ characterization through measurements is also reported. In the proposed model, an EC is derived from the device structure, in terms of electrical lumped elements. Each electrical element in the EC is modeled with an ANN. Then these ANNs are connected together as per the EC and implemented in Verilog-A. The proposed model performance is validated by comparing the circuit simulation results with the measured response of a simple common-source amplifier, which has shown 12.2 $\mathrm{dB}$ gain, $50 \mu \mathrm{W}$ power consumption and $85 \mathrm{kHz} 3-\mathrm{dB}$ frequency with a power supply of $6 \mathrm{~V}$. The same circuit is tested as an inverter and its response is also presented up to $50 \mathrm{kHz}$, from both simulations and measurements. These results show that the model is capable of capturing both small and large signal behavior of the device to good accuracy, even including the harmonic distortion of the signal (that emphasizes the nonlinear behavior of the parasitic capacitance), making the model suitable for IC design.
\end{abstract}

Pydi Bahubalindrun

p.bahubalindruni@campus.fct.unl.pt

1 CENIMAT/I3N, Departamento de Ciência dos Materiais, Faculdade de Ciências e Tecnologia, FCT, Universidade Nova de Lisboa and CEMOP-UNINOVA, 2829-516 Caparica, Portugal

2 INESC TEC and Faculty of Engineering, University of Porto, Campus FEUP, Rua Dr. Roberto Frias, 378, 4200-465 Porto, Portugal
Keywords Equivalent circuit approach · neural models · Verilog-A $\cdot$ a-IGZO TFT modeling $\cdot$ a-IGZO TFT circuits

\section{Introduction}

In recent years, analog/mixed signal circuit design using a-IGZO TFT technology $[3,13,15]$ is gaining significant interest because of its exceptional characteristics, such as relative high mobility, low-temperature and low-cost fabrication $[4,10,11]$. This demands accurate device models that can predict both static and dynamic behavior of the device during circuit simulations. However, most of the reported circuits are confined to employ either a-Si:H [15] or a-IGZO static models [3, 13] (only with overlap capacitance).

In general, the work reported on a-IGZO TFTs modeling is either from device physics $[6,14]$ or from electrical measurements [3], but most of these models are also confined to static behavior characterization. However, it is essential to have an accurate device model that can predict both static and dynamic behavior. Nevertheless, a device model describing both $\mathrm{I}-\mathrm{V}$ and $\mathrm{C}-\mathrm{V}$ characteristics is presented in [1]. Here, $\mathrm{C}-\mathrm{V}$ is limited to only total gate capacitance $\left(\mathrm{C}_{\mathrm{G}}\right)$ and no details were provided on the individual TFT capacitance components between different electrodes, namely, gate to source $\left(\mathrm{C}_{\mathrm{GS}}\right)$ and gate to drain $\left(\mathrm{C}_{\mathrm{GD}}\right)$, which are essential in circuit design. Another good contribution was given in [12] towards a full description of both components by adapting MOSFET SPICE Level 3 model to the particular case of the a-IGZO TFT. Nevertheless, this model is limited in nature, as it is unable to precisely predict the unique properties of the amorphous oxide semiconductors (AOS) present in the TFT, since it is mainly meant for the crystalline silicon. 
The IGZO TFT technology is still in an emerging phase, experiments are being conducted to optimize the device performance by various means $[5,8]$. In order to facilitate immediate circuit design, accurate device models with small development time are required. Though physical models are recommended for circuit design and statistical analysis, their development time is significantly high. Especially, when the technology is still emerging, it is quite challenging to develop physical models since whenever an effort is made for the device optimization, e.g. changing materials or processing conditions, the corresponding physical phenomena that influence the charge carrier flow should be characterized and modeled. Therefore, this paper proposes an accurate behavioral model using ANN based EC approach that avoids deep theoretical analysis about device conduction mechanisms, and through which a workable model is devised in the order of minutes. In the proposed model EC is derived from the device structure (Fig. 1(a)). Consequently, this model cannot be treated as pure empirical, despite it is being developed solely from the device measured characteristics.

The EC of the device (TFT) consists of parasitic elements between different electrodes and a dependent current source that represents the current between drain and source, as shown in Fig. 1(b). Since the bulk (glass) is an insulator, there are no significant parasitics associated with it and can be neglected as their contribution in determining the dynamic behavior of the transistor is trivial. On the other hand, it is crucial to include device capacitance components between different electrodes $\left(\mathrm{C}_{\mathrm{GD}}\right.$ and $\left.\mathrm{C}_{\mathrm{GS}}\right)$ as Fig. 1(b) shows. Since the proposed model is developed from device measurements, it is essential to extract measured data for both $\mathrm{C}_{\mathrm{GD}}$ and $\mathrm{C}_{\mathrm{GS}}$. Most of the articles reported the total gate capacitance $\left(\mathrm{C}_{\mathrm{G}-\mathrm{DS}}\right)$ of a-IGZO TFTs at different frequencies [7] and different bias voltages [1]. However, to the best of authors knowledge individual intrinsic capacitance $\left(\mathrm{C}_{\mathrm{GSi}}=\mathrm{C}_{\mathrm{GS}}-\mathrm{C}_{\mathrm{OV}-\mathrm{GS}}\right.$ and $\mathrm{C}_{\mathrm{GDi}}=$

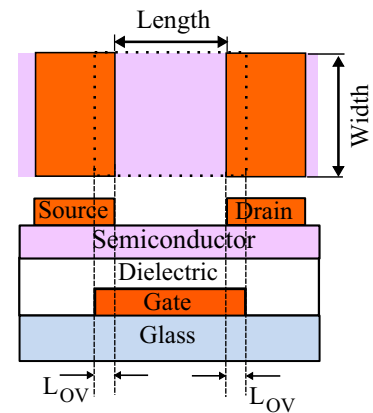

(a)

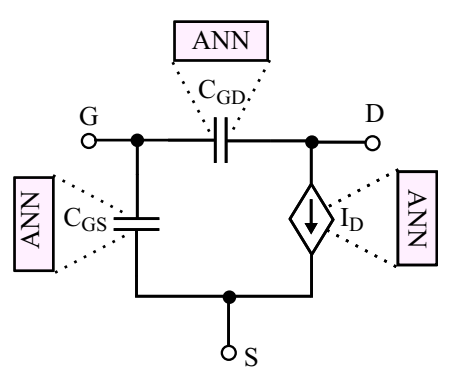

(b)
Fig. 1 a-IGZO TFT a Schematic. b Equivalent circuit of the device, in which individual elements need to be modeled with ANNs
$\mathrm{C}_{\mathrm{GD}}-\mathrm{C}_{\mathrm{OV}-\mathrm{GD}}$ ) characterization and modeling in the complete operating region of the transistor has not yet been addressed in literature for this TFT technology, which is essential for circuit design, in order to predict its frequency dependent behavior. This issue is also addressed in the present paper by characterizing the intrinsic capacitance of the device from measurements.

Once all the measurements are available for the drain current $\left(\mathrm{I}_{\mathrm{D}}\right), \mathrm{C}_{\mathrm{GD}}$ and $\mathrm{C}_{\mathrm{GS}}$, a separate $\mathrm{ANN}$ is developed for each element to achieve good accuracy. Then, the device model is obtained by joining these three ANNs as per the EC. This model is then implemented in Verilog-A for circuit simulations. Later, the model performance is validated through a simple common source (CS) configuration, operating as an amplifier and an inverter (which is a fundamental block in the IC design) by comparing circuit simulation results with the measured response.

Section 2 gives details on the device characterization including capacitance measurements. Section 3 presents the device modeling. Section 4 shows experimental results and discussion and finally main conclusions are drawn in Sect. 5.

\section{Device characterization}

In this work, device static and dynamic characterization is done through measurements as a first step. Devices used for this end are similar to the ones reported in [3], i.e., staggered bottom gate IGZO TFTs with $\mathrm{Ta}_{2} \mathrm{O}_{5}$ - based multilayer/multicomponent dielectric, annealed at $200^{\circ} \mathrm{C}$. I-V and $\mathrm{C}-\mathrm{V}$ characteristics are measured with Keithley 4200-SCS parameter analyzer at room temperature. Figure 2 presents the transfer characteristics of the TFT with $\mathrm{W}=640 \mu \mathrm{m}$ and $\mathrm{L}=20 \mu \mathrm{m}$. These devices are showing a turn-on voltage $\left(\mathrm{V}_{\mathrm{on}}\right)$ of $-0.7 \mathrm{~V}$, a threshold voltage $\left(\mathrm{V}_{\mathrm{TH}}\right)$ of $\sim 1.35 \mathrm{~V}$, on-off ratio greater than $10^{7}$ and mobility

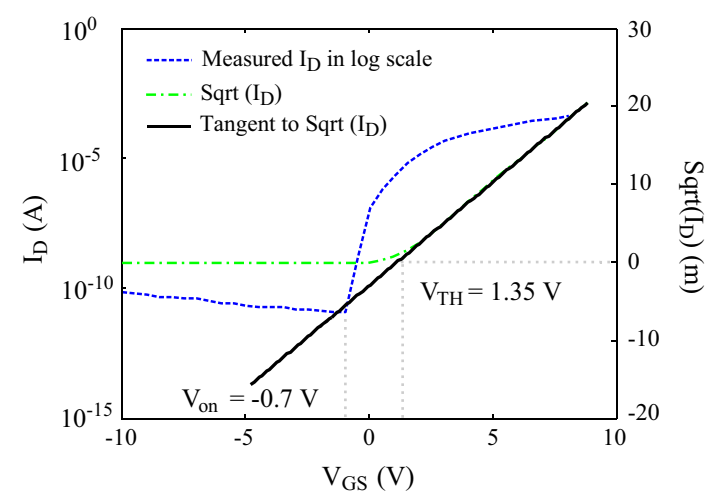

Fig. 2 IGZO TFT transfer characteristics showing $\mathrm{V}_{\text {on }}=-0.7 \mathrm{~V}$ and $\mathrm{V}_{\mathrm{TH}} \sim 1.35 \mathrm{~V}$ 


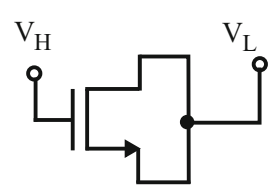

(a)

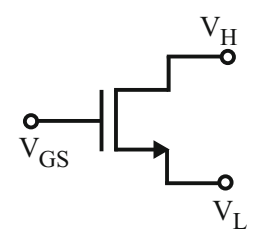

(b)

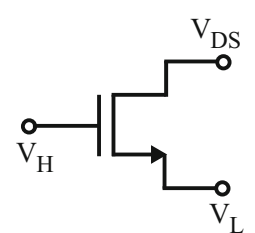

(c)
Fig. 3 Test setup to measure capacitance between different electrodes of a device: a $\mathrm{C}_{\mathrm{G}-\mathrm{Ds}}$. b $\mathrm{C}_{\mathrm{GD}}$. $\mathbf{c} \mathrm{C}_{\mathrm{GS}}+\mathrm{C}_{\mathrm{GD}}$

around $20 \mathrm{~cm}^{2} / \mathrm{V}$ s. All the devices in this work have a gate to drain/source overlap of $5 \mu \mathrm{m}$ and a channel length of 20 $\mu \mathrm{m}$.

\subsection{Capacitance measurements}

The total gate capacitance $\left(\mathrm{C}_{\mathrm{G}-\mathrm{DS}}\right)$ of the TFT is measured with the test setup shown in Fig. 3(a). In this measurement, the source and the drain of the TFT are electrically shorted, and connected to the ground terminal $\left(\mathrm{V}_{\mathrm{L}}\right)$ while the gate is connected to the high terminal $\left(\mathrm{V}_{\mathrm{H}}\right)$ of the Capacitance Voltage Measurement Unit (CVU). A dc voltage sweep of -6 to $6 \mathrm{~V}$ in steps of $0.5 \mathrm{~V}$ is applied to the gate, with an ac signal of $100 \mathrm{mV}$ rms superimposed to the bias. The $\mathrm{C}-\mathrm{V}$ measurements are repeated for different frequencies ranging from 30 to $500 \mathrm{kHz}$. The resulted $\mathrm{C}-\mathrm{V}$ characteristics for a TFT wtih $\mathrm{W}=640 \mu \mathrm{m}$ is shown in Fig. 4. From these measurements it can be noticed that the total overlap capacitance $\left(\mathrm{C}_{\mathrm{OV}-\mathrm{GS}}+\mathrm{C}_{\mathrm{OV} \text {-GD }}\right)$ due to the overlap between gate to drain/source electrodes is $\sim 3.7 \mathrm{pF}$ and the channel capacitance $\left(\mathrm{C}_{\mathrm{CH}}=\mathrm{C}_{\mathrm{G}}\right.$-overlap capacitance $) \sim 3.3 \mathrm{pF}$. It can also be noticed that the measured capacitance is almost independent of the input signal frequency, at least within the applied signal frequency range. Therefore, for the other $\mathrm{C}-\mathrm{V}$ measurements, an input ac signal with $30 \mathrm{kHz}$ frequency is applied.

$\mathrm{C}_{\mathrm{GD}}$ can be directly measured with the test setup shown in Fig. 3(b), where a dc bias is applied at the gate and signal is applied at the drain. Under this test setup, the gate acts as ground for signal, allowing for direct measurement of $\mathrm{C}_{\mathrm{GD}}$. On the other hand, Fig. 3(c) determines $\mathrm{C}_{\mathrm{GS}}+$ $\mathrm{C}_{\mathrm{GD}}$, as drain acts as ground for signal. By subtracting $\mathrm{C}_{\mathrm{GD}}$ from these values, $\mathrm{C}_{\mathrm{GS}}$ can be determined. Intrinsic capacitances $\left(\mathrm{C}_{\mathrm{GSi}}\right.$ and $\left.\mathrm{C}_{\mathrm{GD}}\right)$ between different electrodes of the device are obtained by subtracting the overlap capacitance from $\mathrm{C}_{\mathrm{GS}}$ and $\mathrm{C}_{\mathrm{GD}}$. Figure 5 shows the respective measurement results, revealing a close agreement with Meyers FET capacitive model [9] i.e., in the linear region $\mathrm{C}_{\mathrm{GSi}}=\mathrm{C}_{\mathrm{GDi}} \sim \frac{1}{2} \mathrm{C}_{\mathrm{CH}}$ and in the deep saturation, $\mathrm{C}_{\mathrm{GSi}} \sim \frac{2}{3} \mathrm{C}_{\mathrm{CH}}$ and $\mathrm{C}_{\mathrm{GDi}}$ approaches to 0 .

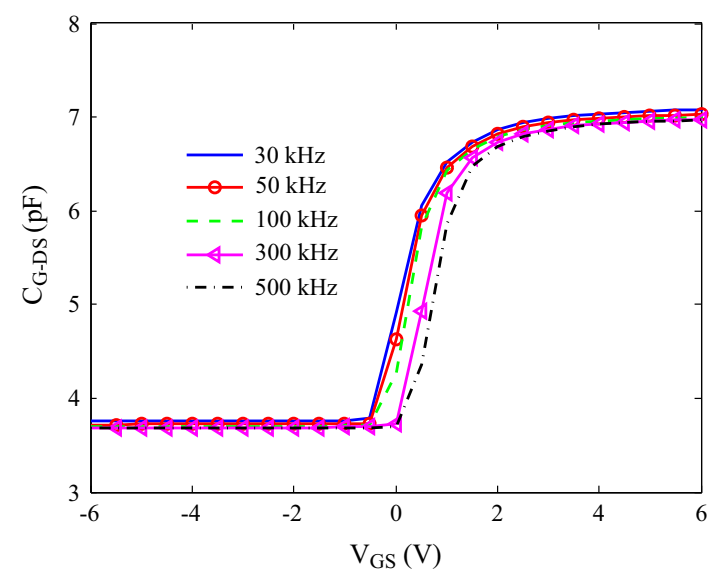

Fig. 4 Measured $\mathrm{C}_{\mathrm{G}-\mathrm{DS}}$ at various frequencies, for a gate voltage sweep from -6 to $6 \mathrm{~V}$ in steps of $0.5 \mathrm{~V}$

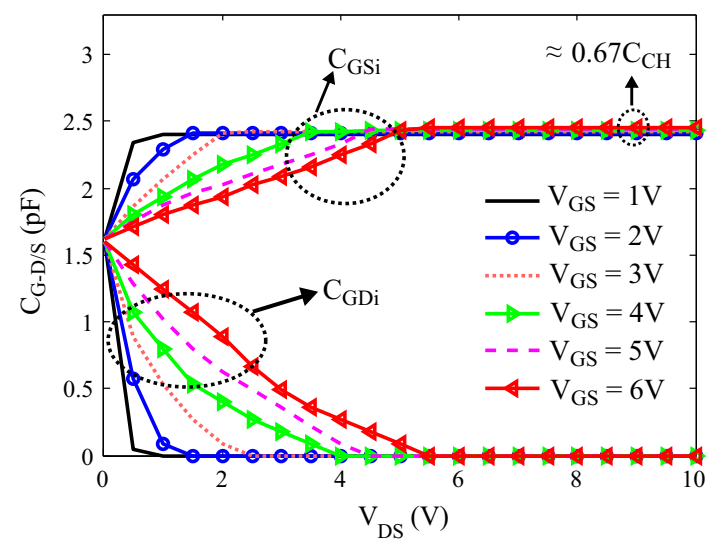

Fig. 5 Measured $\mathrm{C}_{\mathrm{GSi}}$ and $\mathrm{C}_{\mathrm{GDi}}$ for varying bias voltages

\section{Device model}

ANNs are good candidates for function estimation problems. ANN architecture corresponds to that of a parallel computing machine, formed from the interconnection of a set of artificial neurons. Each neuron in the network, commonly known as processing element, has a set of inputs, synaptic weights and bias. Training samples are applied at the input layer of the network so that it can learn the underlying physical process, subjected to a cost-function minimization. Single hidden layer multilayer perceptron (MLP) networks trained with backpropagation algorithm are used in this work. More details of the MLP modeling approach can be found in [2,3].

The proposed method uses a separate ANN for each element of the EC as shown in Fig. 1(b) to guarantee a continuous model with good accuracy. Once they promise good accuracy, all the ANNs (for $\mathrm{I}_{\mathrm{D}}, \mathrm{C}_{\mathrm{GD}}$ and $\mathrm{C}_{\mathrm{GS}}$ ) are implemented in Verilog-A to realize the EC that can 
predict the static and dynamic behavior of the device during circuit simulations.

\section{Experimental results and discussion}

First, $\mathrm{I}_{\mathrm{D}}, \mathrm{C}_{\mathrm{GDi}}$ and $\mathrm{C}_{\mathrm{GSi}}$ are obtained from the developed model at various bias voltages, from the Cadence Spectre circuit simulator. They show good agreement $(<5 \%$ mean absolute relative error) with the corresponding measurements, as it can be observed from Fig. 6 .

Then this model is used to simulate the circuit, whose schematic and micrograph of the fabricated circuit (which is fabricated with the same conditions as the TFTs that are used to develop the model) with wire bonding are shown in Fig. 7. The resistor $(\mathrm{R})$ is a discrete/external component to the circuit, allowing for increased flexibility during testing.

Small signal high-frequency model of the circuit is shown in Fig. 8. By applying KCL at node $\mathrm{v}_{\mathrm{x}}$,

$$
\begin{array}{r}
\frac{v_{x}-v_{\text {in }}}{R}+s c_{g s 1} v_{x}+s c_{g d 1}\left(v_{x}-v_{\text {out }}\right)=0 \\
v_{x}=\frac{v_{\text {in }}+s R c_{g d 1} v_{\text {out }}}{1+s R\left(c_{g s 1}+c_{g d 1}\right)}
\end{array}
$$

similarly at node $\mathrm{v}_{\text {out }}$ :

$$
\begin{array}{r}
s c_{\text {gd } 1}\left(v_{\text {out }}-v_{x}\right)+g_{m 1} v_{x}+\frac{v_{\text {out }}}{r_{o 1}}+s c_{\text {gs } 2} v_{\text {out }} \\
+g_{m 2} v_{\text {out }}+\frac{v_{\text {out }}}{r_{\text {o } 2}}+s c_{L} v_{\text {out }}=0
\end{array}
$$

(1) and (2) results in

$$
\begin{aligned}
A_{v} & =\frac{v_{\text {out }}}{v_{\text {in }}}=\frac{g_{m 1}-s c_{\text {gd } 11}}{A s^{2}+B s+C} \\
& =\frac{g_{m 1}-s c_{g d 1}}{\left(1+\frac{\omega}{p 1}\right)\left(1+\frac{\omega}{p 2}\right)}
\end{aligned}
$$

where

$$
\begin{aligned}
& A=R\left[c_{g d 1}\left(c_{g s 1}+c_{g s 2}+c_{L}\right)+c_{g s 1}\left(c_{g s 2}+c_{L}\right)\right] \\
& B=R\left[g_{m 1} c_{g d 1}+g_{m 2 t}\left(c_{g s 1}+c_{g d 1}\right)\right]+c_{g d 1}+c_{g s 2}+c_{L} \\
& C=g_{m 2}+g_{d s 1}+g_{d s 2}
\end{aligned}
$$

Therefore the circuit has two poles (p1 and $\mathrm{p} 2$ ) and a single zero $\left(\frac{g_{m 1}}{2 \pi c_{g d 1}}\right)$. When $\mathrm{R}$ is made to zero, the amplifier reduces to single pole system. In this case the amplifier gain $\left(A_{v}\right)$ is given by

$A_{v}=\frac{v_{\text {out }}}{v_{\text {in }}}=\frac{g_{m 1}-s c_{g d 1}}{s\left(c_{g d 1}+c_{g s 2}+c_{L}\right)+g_{m 2}+g_{d s 1}+g_{d s 2}}$

Therefore the pole is given by,

$p 1=-\frac{g_{m 2}+g_{d s 1}+g_{d s 2}}{2 \pi\left(c_{g d 1}+c_{g s 2}+c_{L}\right)}$

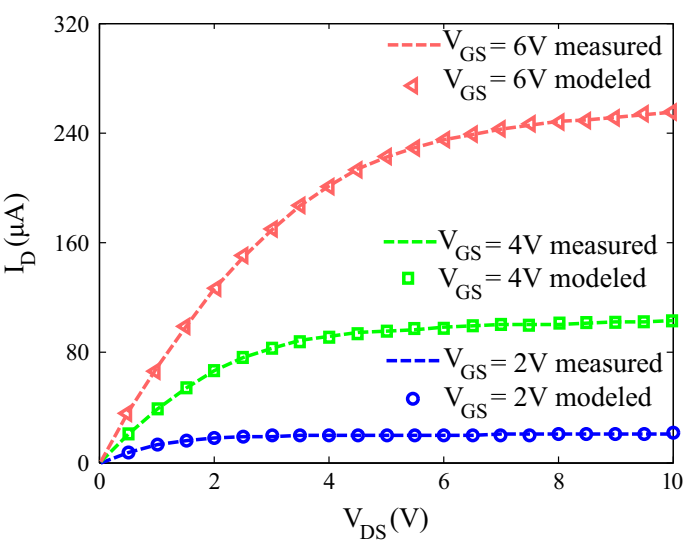

(a)

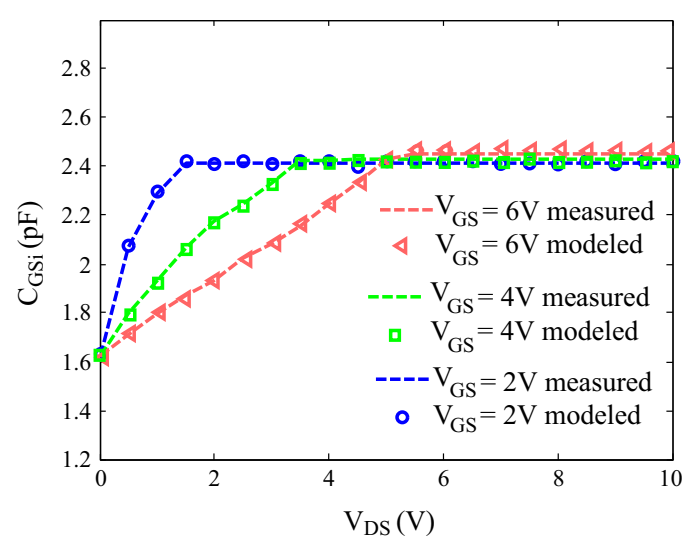

(b)

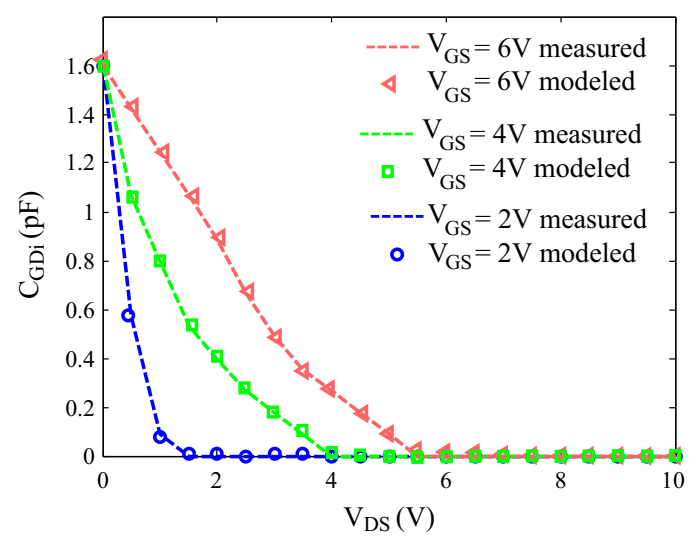

(c)

Fig. 6 Verilog-A neural model performance validation from the measurements $0 \leq \mathrm{V}_{\mathrm{DS}} \leq 10 \mathrm{~V}$ in steps of $0.5 \mathrm{~V}$ and $2 \leq \mathrm{V}_{\mathrm{GS}} \leq$ $6 \mathrm{~V}$ in steps of $2 \mathrm{~V}$ : $\mathbf{a} \mathrm{I}_{\mathrm{D}} \cdot \mathbf{b} \mathrm{C}_{\mathrm{GSi}} \cdot \mathbf{c} \mathrm{C}_{\mathrm{GDi}}$

and the low frequency small signal gain is given by,

$$
-\frac{g_{m 1}}{g_{m 2}+g_{d s 1}+g_{d s 2}}
$$

This circuit simulations and measurements were carried out with a $4 \mathrm{pF}$ load and a power supply $\left(\mathrm{V}_{\mathrm{DD}}\right)$ of $6 \mathrm{~V}$. 


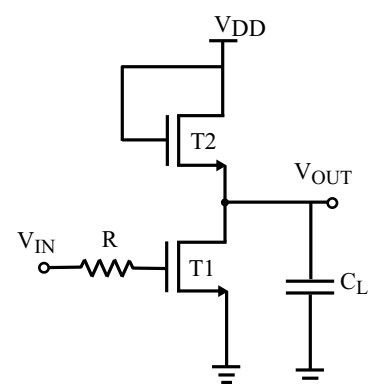

(a)

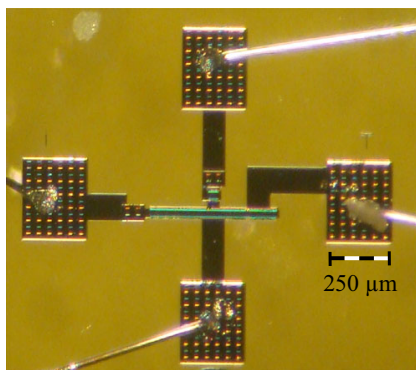

(b)
Fig. 7 CS amplifier a schematic- $\mathrm{W}_{(\mathrm{T} 1)}=640 \mu \mathrm{m}$ and $\mathrm{W}_{(\mathrm{T} 2)}=40$ $\mu \mathrm{m}$. b Fabricated circuit micrograph after wire bonding

Measurements were performed under normal ambient at room temperature with the help of AFG3021B single channel arbitrary function generator and DS2024B four channel digital storage oscilloscope. Frequency response of the circuit is shown in Fig. 9 with different values of $\mathrm{R}(0 \Omega, 100$ $\mathrm{K} \Omega$ and $1 \mathrm{M} \Omega$ ). Corresponding dominant pole is presented for each case in Table 1, both from simulations and measurements, which is also well predicted by (3) and (4).

During amplifier characterization from measurements, care has been taken to keep all the TFTs in saturation. This amplifier resulted in $12.2 \mathrm{~dB}$ gain as predicted by (7). It also has shown a very low power consumption of $50 \mu \mathrm{W}$ and $85 \mathrm{kHz} 3-\mathrm{dB}$ frequency. Then, the circuit is characterized without $\mathrm{R}$, for a sinusoidal input signal with 100 $\mathrm{kHz}$ frequency. Figure 10 shows the output signal and error (difference between the simulation and measured output) signal, which is showing $2.5 \%$ mean absolute relative error. Corresponding THD from simulation and measurements are 14 and $11.2 \%$, respectively.

Later, the same circuit is tested as a logic inverter. When the input voltage $\left(\mathrm{V}_{\mathrm{N}}\right)$ is set to a low value, $\mathrm{T} 1$ is almost turned off and nearly no current flows through the device. Consequently, the output voltage $\left(\mathrm{V}_{\text {OUT }}\right)$ approaches to $\mathrm{V}_{\mathrm{DD}}-\mathrm{V}_{\mathrm{TH}}$, because of the diode connected load. As $\mathrm{V}_{\mathrm{IN}}$ gets higher, the drain current increases thus the voltage drops across $\mathrm{T} 2$, which results in a lower $\mathrm{V}_{\text {OUT. }}$. Figure 11 shows the transient response to a input square wave for three different frequencies. In can be noticed that output

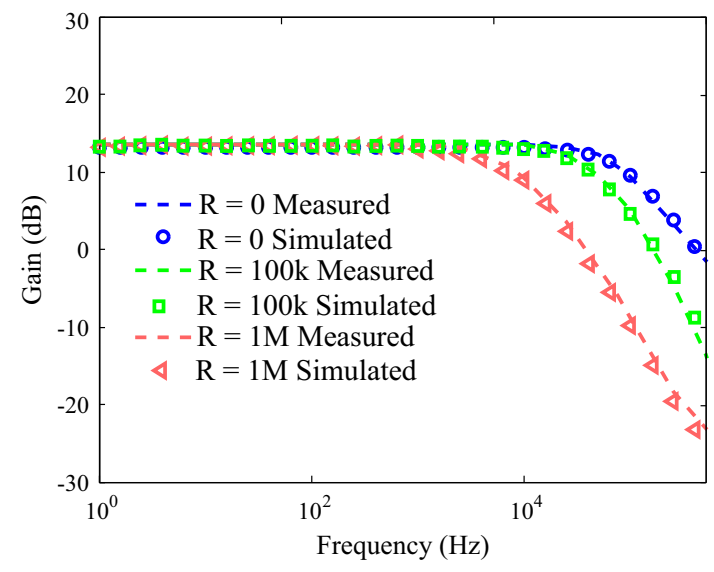

Fig. 9 CS amplifier frequency response

Table 1 Dominant pole locations

\begin{tabular}{llll}
\hline & \multicolumn{2}{l}{ Frequency $(\mathrm{kHz})$} \\
\cline { 2 - 4 } & $\mathrm{R}=0$ & $\mathrm{R}=100 \mathrm{~K}$ & $\mathrm{R}=1 \mathrm{M}$ \\
\hline Simulation (EC) & 84 & 44 & 7.5 \\
Measurement & 85 & 45 & 8 \\
\hline
\end{tabular}

high level amplitude is $\mathrm{V}_{\mathrm{DD}}-\mathrm{V}_{\mathrm{TH}}(=4.65 \mathrm{~V})$ and low level is $\sim 0.15 \mathrm{~V}$. In addition, small peaks can be distinguished in the output waveform in instants of time where the input transitions occur, mainly visible with the high frequency $(50 \mathrm{kHz})$ signal. This is caused by charge injection from the transistor gate capacitance. Both load and transistor capacitors are responsible for the rise and fall times. The load transistor possesses less current capability (higher equivalent resistance) while the driver transistor (T1) has much higher current driving capabilities (wider transistor), which justifies the much higher rise time than the fall time. Such is a consequence of a design that favours some gain $\left(\sqrt{\frac{\mathrm{W} 1}{\mathrm{~W} 2}}\right)$ in detriment of symmetric swings, as it can be noticed from Fig. 11.

In all the circuits measurements, as the load $(4 \mathrm{pF})$ is a reasonable value compared to the device intrinsic capacitances, measurements can really elevate the proposed
Fig. 8 small signal high frequency model of the CS amplifier including the load capacitor

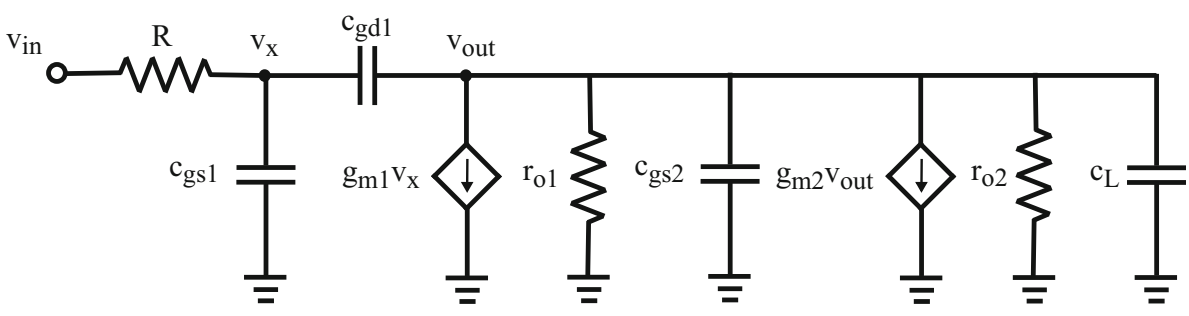




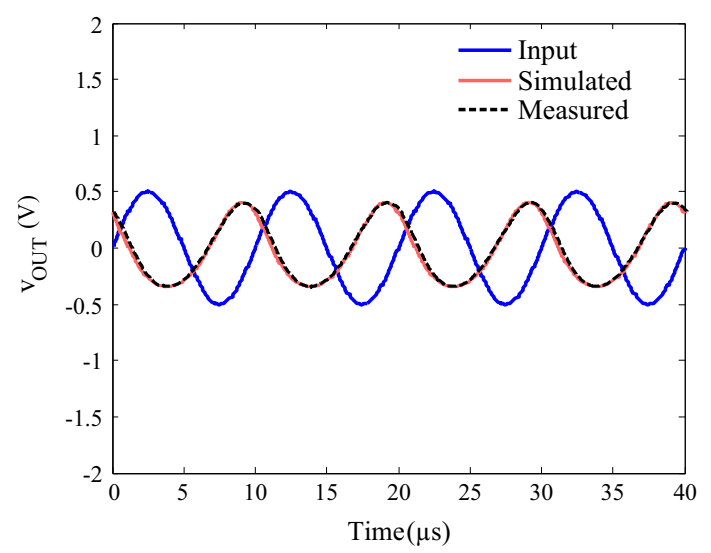

(a)

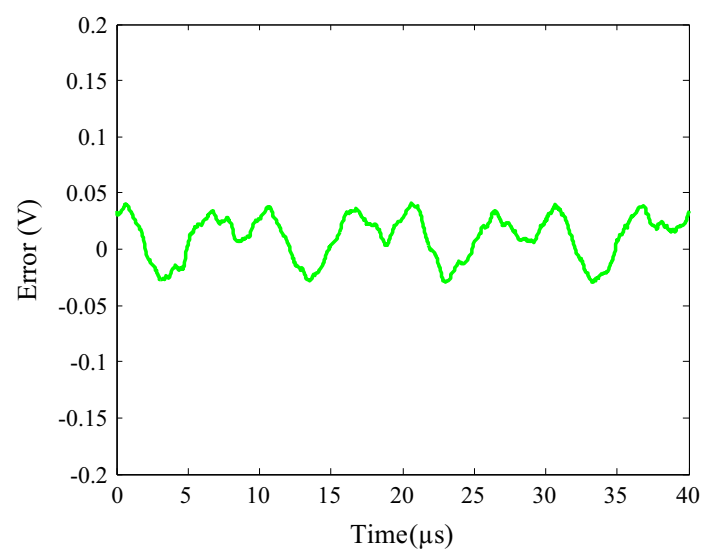

(b)

Fig. 10 CS amplifier response form simulations and measurements, when $\mathrm{f}$ is $100 \mathrm{kHz}$. a Output. b Error signal

model accuracy for both static and dynamic characterization. All the above results demonstrate the model ability to predict the small and large-signal behavior including nonlinear effects of the parasitic capacitance from the circuit response. Circuit bandwidth can be improved by scaling down the devices (considering small lengths) and using self aligned structure (to minimize overlap capacitance).

\section{Conclusions}

This paper proposed an accurate behavioral model for the a-IGZO TFT using ANN based EC approach and implemented in Verilog-A for circuit simulations. Intrinsic capacitance of the device is characterized through measurements. Circuits simulations from the developed model have shown a good agreement with the corresponding measurements that demonstrates the modeling ability in predicting device static and dynamic behavior including non-linear distortion.
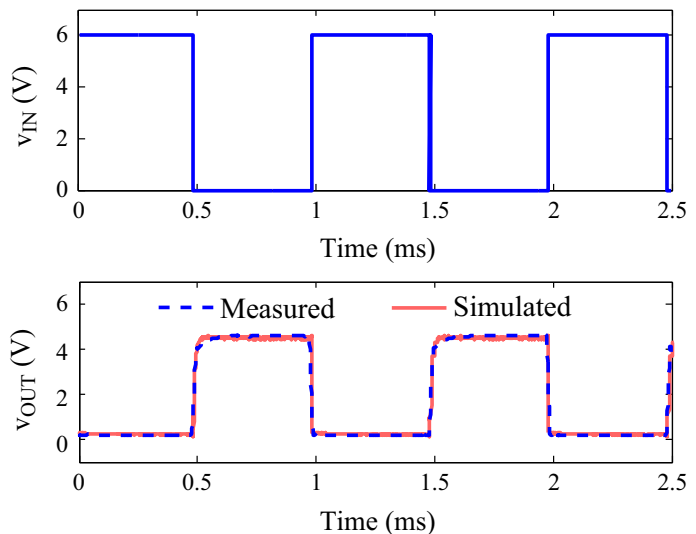

(a)
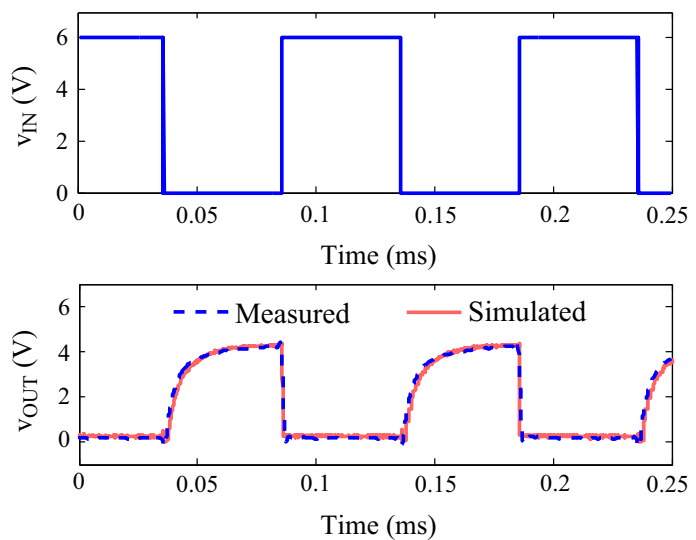

(b)
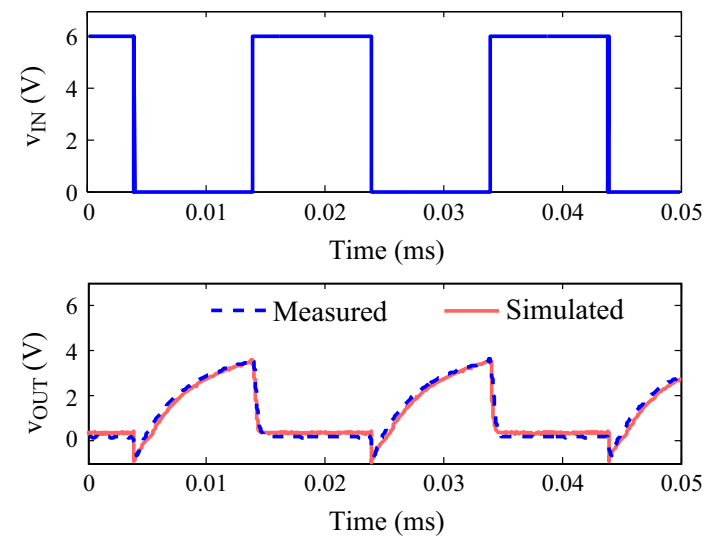

(c)

Fig. 11 Circuit transient response form simulations and measurements, when the input signal frequency is a $1 \mathrm{kHz}$. b $10 \mathrm{kHz}$. c 50 $\mathrm{kHz}$

Acknowledgments This work is funded by FEDER funds through the COMPETE 2020 Programme and National Funds through FCTPortuguese Foundation for Science and Technology under the Project Nos. CMUPT/SIA/0005/2009, UID/CTM/50025/2013 and EXCL/ CTM-NAN/0201/2012. The work has also received funding from the European Communities 7th Framework Programme under grant 
agreement ICT-2013-10-611070 (i-FLEXIS project) and from H2020 program under ICT-03-2014-644631 (ROLL-OUT project).

\section{References}

1. Bae, M., Kim, Y., Kong, D., Jeong, H. K., Kim, W., Kim, J., et al. (2011). Analytical models for drain current and gate capacitance in amorphous InGaZnO thin-film transistors with effective carrier density. Electron Device Letters, 32(11), 1546-1548.

2. Bahubalindruni, G., Tavares, V. G., Barquinha, P., Duarte, C., Martins, R., Fortunato, E., de Oliveira, P. G. (2012). Basic analog circuits with a-GIZO thin-film transistors: Modeling and simulation. In $S M A C D$.

3. Bahubalindruni, P. G., Grade Tavares, V., Barquinha, P., Duarte, C., Guedes de Oliveira, P., Martins, R., et al. (2013). Transparent current mirrors with a-GIZO TFTs: Neural modeling, simulation and fabrication. Journal of Display Technology, 9(12), 1001-1006.

4. Barquinha, P., Pereira, L., Goncalves, G., Martins, R., \& Fortunato, E. (2008). The effect of deposition conditions and annealing on the performance of high-mobility GIZO TFTs. Electrochemical and Solid-State Letters, 11(9), 248-251.

5. Chou, K.-I., Hsu, H.-H., Cheng, C.-H., Lee, K.-Y., Li, S.-R., \& Chin, A. (2013). A low operating voltage IGZO TFT using $\mathrm{LaLuO} 3$ gate dielectric. In IEEE international conference of electron devices and solid-state circuits (EDSSC) (pp. 1-2).

6. Deng, W., Huang, J., Ma, X., \& Ning, T. (2014). An explicit surface-potential-based model for amorphous IGZO thin-film transistors including both tail and deep states. Electron Device Letters, 35(1), 78-80.

7. Lee, S., Park, S., Kim, S., Jeon, Y., Jeon, K., Park, J. H., et al. (2010). Extraction of subgap density of states in amorphous InGaZnO thin-film transistors by using multifrequency capacitance-voltage characteristics. Electron Device Letters, 31(3), 231-233.

8. Li, X., Geng, D., Mativenga, M., \& Jang, J. (2014). High-speed dual-gate a-igzo tft-based circuits with top-gate offset structure. IEEE Electron Device Letters, 35(4), 461-463.

9. Meyer, J. E. (1971). Mos models and circuit simulation. RCA Review, 32, 42-63.

10. Nomura, K., Ohta, H., Takagi, A., Kamiya, T., Hirano, M., \& Hosono, H. (2004). Room-temperature fabrication of transparent flexible thin-film transistors using amorphous oxide semiconductors. Nature, 432(7016), 488-492.

11. Olziersky, A., Barquinha, P., Vila, A., Magana, C., Fortunato, E., Morante, J., et al. (2011). Role of $\mathrm{Ga}_{2} \mathrm{O}_{3}-\mathrm{In}_{2} \mathrm{O}_{3}-\mathrm{ZnO}$ channel composition on the electrical performance of thin-film transistors. Materials Chemistry and Physics, 131(1), 512-518.

12. Perumal, C., Ishida, K., Shabanpour, R., Boroujeni, B. K., Petti, L., Munzenrieder, N. S., et al. (2013). A compact a-IGZO TFT model based on MOSFET SPICE level=3 template for analog/RF circuit designs. Electron Device Letters, 34(11), 1391-1393.

13. Raiteri, D., Torricelli, F., Myny, K., Nag, M., van der Putten, B., Smits, E., Steudel, S., Tempelaars, K., Tripathi, A., Gelinck, G., van Roermund, A., Cantatore, E. (2012) A 6b 10MS/s currentsteering DAC manufactured with amorphous Gallium-IndiumZinc-Oxide TFTs achieving SFDR $>30 \mathrm{~dB}$ up to $300 \mathrm{kHz}$. In ISSCC (pp. 314-316).

14. Tsormpatzoglou, A., Hastas, N. A., Choi, N., Mahmoudabadi, F., Hatalis, M. K., \& Dimitriadis, C. A. (2013). Analytical surfacepotential-based drain current model for amorphous ingazno thin film transistors. Journal of Applied Physics, 114(18), 184502-1845026.
15. Zysset, C., Munzenrieder, N., Petti, L., Buthe, L., Salvatore, G. A., \& Troster, G. (2013). IGZO TFT-based all-enhancement operational amplifier bent to a radius of $5 \mathrm{~mm}$. Electron Device Letters, 34(11), 1394-1396.

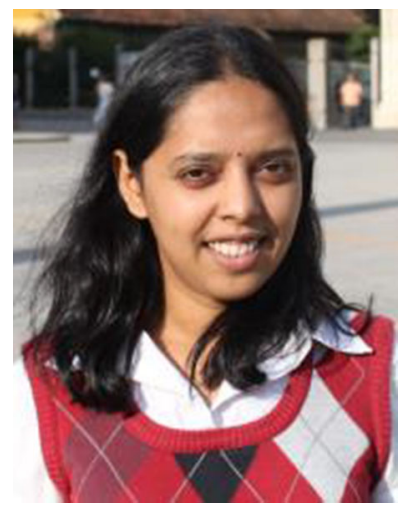

Pydi Bahubalindruni received B.Tech degree in Electronics and Communications and M.Tech degree in Electronic Instrumentation from JNTU and NIT Warangal in India, respectively. She obtained her Ph.D. degree from FEUP/INESC in Department of Electrical and Computer Engineering. Currently she is a postdoctoral researcher at CENIMAT, FCTUNL. Her research interests include device characterization, modeling and analog/mixed signal circuits design with emerging technologies.

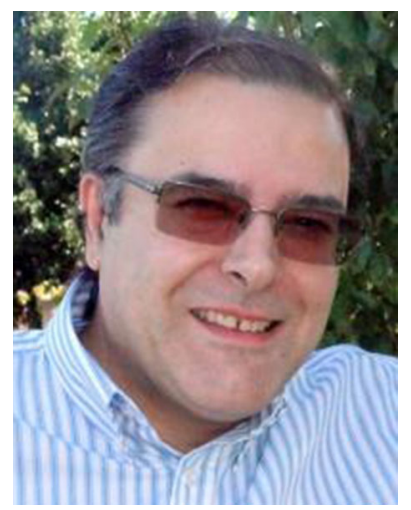

Vítor Grade Tavares received the Licenciatura and M.S. degrees in electrical engineering from the University of Aveiro, Portugal, in 1991 and 1994. He concluded his Ph.D. degree in electrical engineering, from the Computational NeuroEngineering Laboratory at the University of Florida, Gainesville, USA, in 2001. In 1999 he joined the University of Porto, Portugal, as an Invited Assistant, where he has been an Assistant Professor since 2002. He is also a Senior Researcher at the INESC Porto. His research interests include low power, mixed signal and neuromorphic integrated-chip design and biomimetic computing, CMOS RF integrated circuit design and transparent electronics. In 2010 he was a Visiting Professor at Carnegie Mellon University, USA, and was a co-founder and chairman of the Portuguese IEEE Education Society Chapter.

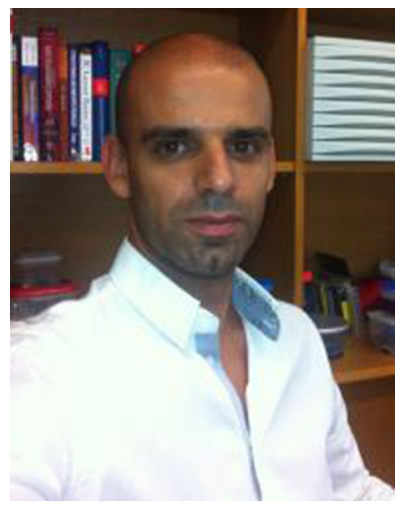

Pedro Barquinha received the $\mathrm{Ph} . \mathrm{D}$. degree from Universidade Nova de Lisboa in 2010, in Nanotechnologies and Nanosciences, with the dissertation "Transparent Oxide Thin-Film Transistors". He is an Assistant Professor at the Materials Science Department of FCT-UNL since 2012. His work in transparent electronics spans many areas, from the design, deposition and characterization of multicomponent oxides, fabrication and characterization of oxide TFTs, to their integration in analog and digital circuits on flexible substrates. He is co-author of 98 peer-reviewed papers, with more than 3800 citations (h-index $=30$, as January 2016). He co-authored 2 books and 2 book chapters on this area and gave more than 
30 invited lectures including 2 key-notes in international scientific conferences and workshops. His Figacurrent research focus is to take transparent electronics to performance and integration levels suitable for future ICTs. This involves pursuing reproducible and low temperature synthesis routes of high quality oxide nanostructures and integrating them in nanodevices and circuits, always complemented by device modeling/simulation.

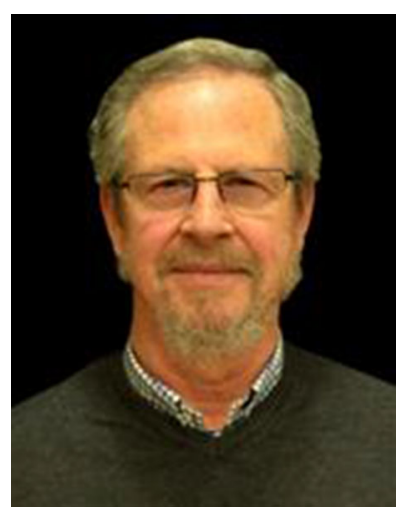

Pedro Guedes de Oliveira is Retired Professor of Electronics at the Faculty of Engineering of the University of Porto (FEUP) and his research interests are in the area of electronic circuits and microelectronics, signal processing and biomedical applications. He was formerly President of INESC Porto and head of Electrical and Computer Engineering Department of FEUP.

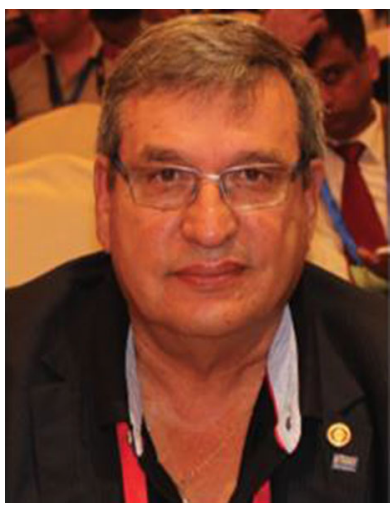

Rodrigo Martins Portuguese, Full Professor of Faculty of Sciences and Technology of New University of Lisbon, Portugal, in the field of Advanced Functional Materials and Microelectronics. Head of Materials Science Department, member of the Portuguese Academy of Engineering and of the Portuguese Order of Engineers. Immediate Past President of the European Materials Research Society (E-MRS) and running president of the senate of E-MRS. Founder and director of the Centre of Excellence in Microelectronics and Optoelectronics Processes of Uninova; leader of the Materials, Optoelectronics and Nanotechnologies group of I3N/
CENIMAT. Expert in the field of transparent electronics and on the paper electronics Member of the: advisory board of Horizon 2020 (Advanced Materials, Nanotechnologies, Biotechnologies and Manufacturing); International Advisory Board of Strategic Initiative Materials, a Flanders industry initiative; International Advisory Quality Assurance Board of the ERASMUS MUNDUS MASTER COURSE FAME (Functionalized Advanced Materials and Engineering); Steering Committee of EuMat; Alliance for Materials; Joint Innovation Centre for Advanced Material, Sino-Portuguese; External Advisory board and steering committee of EU KNIGHTS. Coordinator of the Portuguese $\mathrm{PhD}$ AdvaMTech program; member of the GTA group of the Portuguese Foundation for Science and Technology.

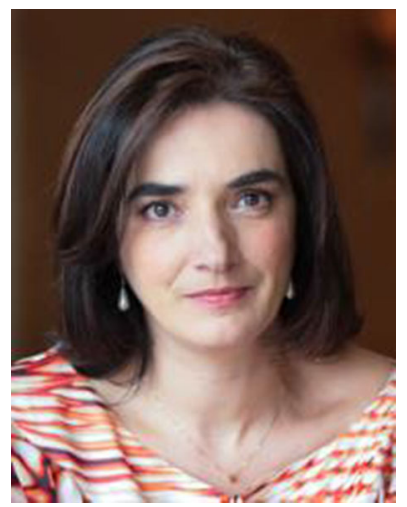

Elvira Fortunato is Full Professor of Materials Science at the Faculty of Sciences and Technology of Universidade Nova de Lisboa, since 2012, specialized in Microelectronics and Nanotechnologies. Presently she is Director of the i3N and CENIMAT and Deputy chair of the Scientific Adviser Mechanism of the Presidency of the European Commission. She pioneered European research on transparent electronics, namely thin-film transistors based on oxide semiconductors, demonstrating that oxide materials may be used as true semiconductors as well as co-inventor of the paper transistor and electrochromic transistor. She is also Director of the $\mathrm{PhD}$ program in Micro and Nanotechnologies Engineering; Associate editor of Pysica Status Solidi Rapid Research Letters, Wiley; CoEditor of Europhysics Letters; Member of the Advisory Editorial Board of Applied Surface Science; Member of the National Scientific and Technological Council and Member of "Academia de Engenharia", Portugal. She filled 68 international patents and got more than 15 international prizes for her work. In 2008 she wins an Advanced Grant from ERC for the project "Invisible" (first edition and she got the first position), which was also considered by ERC as a success story. 